General Mathematics Vol. 27, No. 2 (2019), 3-11

DOI:10.2478/gm-2019-0010

Sciendo

\title{
Two New Classes of Analytic Functions Defined by Strong Differential Subordinations and Superordinations ${ }^{1}$
}

\author{
Abbas Kareem Wanas
}

\begin{abstract}
In the present investigation, by making use of strong differential subordinations and superordinations, we introduce and study two new classes of holomorphic functions containing generalized differential operator. Also we determine important properties for functions belongs to these classes.
\end{abstract}

2010 Mathematics Subject Classification: 30C45, 30A20, 34A40.

Key words and phrases: Holomorphic function, Convex function, Strong Differential Subordinations and Superordinations, Dominant, Subordinant, Generalized differential operator.

\footnotetext{
${ }^{1}$ Received 4 June, 2019

Accepted for publication (in revised form) 8 December, 2019
} 


\section{Introduction}

Indicate by $\mathcal{H}(\mathbb{U} \times \overline{\mathbb{U}})$ the class of holomorphic functions in $\mathbb{U} \times \overline{\mathbb{U}}$, where $\mathbb{U}$ the open unit disk of the complex plane $\mathbb{U}=\{z \in \mathcal{C}:|z|<1\}$, $\mathbb{U}=$ $\{z \in \mathcal{C}:|z| \leq 1\}$ the closed unit disk of the complex plane.

For $n \in \mathbb{N}=\{1,2, \cdots\}$ and $a \in \mathcal{C}$, let $\mathcal{H}[a, n, \varsigma]=\{f \in \mathcal{H}(\mathbb{U} \times \overline{\mathbb{U}})$ : $\left.f(z, \varsigma)=a+a_{n}(\varsigma) z^{n}+a_{n+1}(\varsigma) z^{n+1}+\ldots, z \in \mathbb{U}, \varsigma \in \overline{\mathbb{U}}\right\}$, where $a_{j}(\varsigma)$ are holomorphic functions in $\overline{\mathbb{U}}$ for $j \geq n$.

Let $\mathcal{A}_{\varsigma}$ stands for the class of functions of the form:

$$
f(z, \varsigma)=z+\sum_{k=2}^{\infty} a_{k}(\varsigma) z^{k}, \quad(z \in \mathbb{U}, \varsigma \in \overline{\mathbb{U}})
$$

which are holomorphic in $\mathbb{U} \times \overline{\mathbb{U}}$ and $a_{k}(\varsigma)$ are holomorphic functions in $\overline{\mathbb{U}}$ for $k \geq 2$.

Definition 1 [9]. Let $Q_{\varsigma}$ be the family of all functions that are holomorphic and injective on $\overline{\mathbb{U}} \times \overline{\mathbb{U}} \backslash \mathcal{E}(f, \varsigma)$, where

$$
\mathcal{E}(f, \varsigma)=\left\{r \in \partial \mathbb{U}: \lim _{z \rightarrow r} f(z, \varsigma)=\infty\right\},
$$

and $f_{z}^{\prime}(r, \varsigma) \neq 0$ for $r \in \partial \mathbb{U} \times \overline{\mathbb{U}} \backslash \mathcal{E}(f, \varsigma)$. The subfamily of $Q_{z}$ with $f(0, \varsigma)=a$ is denoted by $Q_{\varsigma}(a)$.

Definition 2 [9]. Let $f(z, \varsigma), F(z, \varsigma)$ be holomorphic in $\mathbb{U} \times \overline{\mathbb{U}}$. The function $f(z, \varsigma)$ is said to be strongly subordinate to $F(z, \varsigma)$ if there exists a function $w$ holomorphic in $\mathbb{U}$ with $w(0)=0$ and $|w(z)|<1(z \in \mathbb{U})$ such that $f(z, \varsigma)=$ $F(w(z), \varsigma)$ for all $\varsigma \in \overline{\mathbb{U}}$. In such a case we write $f(z, \varsigma) \prec \prec F(z, \varsigma), z \in \mathbb{U}$, $\varsigma \in \overline{\mathbb{U}}$.

\section{Remark 1 [9].}

(i) Since $f(z, \varsigma)$ is holomorphic in $\mathbb{U} \times \overline{\mathbb{U}}$, for all $\varsigma \in \overline{\mathbb{U}}$ and schlicht in $\mathbb{U}$, for all $\varsigma \in \overline{\mathbb{U}}$, Definition (2) is equivalent to $f(0, \varsigma)=F(0, \varsigma)$ for all $\varsigma \in \overline{\mathbb{U}}$ and $f(\mathbb{U} \times \overline{\mathbb{U}}) \subset F(\mathbb{U} \times \overline{\mathbb{U}})$.

(ii) If $f(z, \varsigma)=f(z)$ and $F(z, \varsigma)=F(z)$, the strong subordination becomes the usual notion of subordination. 
If $f(z, \varsigma)$ is strongly subordinate to $F(z, \varsigma)$, then $F(z, \varsigma)$ is strongly superordinate to $f(z, \varsigma)$.

As a dual notion of strong differential subordination, Oros [9] has introduced and developed the notion of strong differential superordinations.

Lemma 1 [8]. Suppose that $h(z, \varsigma)$ be a schlicht function with $h(0, \varsigma)=a$ for all $\varsigma \in \overline{\mathbb{U}}$ and $\gamma \in \mathcal{C} \backslash\{0\}$ with $\operatorname{Re}(\gamma) \geq 0$. If $p \in \mathcal{H}[a, 1, \varsigma]$ and

$$
p(z, \varsigma)+\frac{1}{\gamma} z p_{z}^{\prime}(z, \varsigma) \prec \prec h(z, \varsigma), \quad(z \in \mathbb{U}, \varsigma \in \overline{\mathbb{U}}),
$$

then

$$
p(z, \varsigma) \prec \prec q(z, \varsigma) \prec \prec h(z, \varsigma), \quad(z \in \mathbb{U}, \varsigma \in \overline{\mathbb{U}}),
$$

where $q(z, \varsigma)=\gamma z^{-\gamma} \int_{0}^{z} h(t, \varsigma) t^{\gamma-1} d t$ is convex and it is the best dominant of (2).

Lemma 2 [9]. Suppose that $h(z, \varsigma)$ be a convex function with $h(0, \varsigma)=a$ for all $\varsigma \in \overline{\mathbb{U}}$ and $\gamma \in \mathcal{C} \backslash\{0\}$ with $\operatorname{Re}(\gamma) \geq 0$. If $p \in \mathcal{H}[a, 1, \varsigma] \cap Q_{\varsigma}, p(z, \varsigma)+$ $\frac{1}{\gamma} z p_{z}^{\prime}(z, \varsigma)$ is schlicht in $\mathbb{U} \times \overline{\mathbb{U}}$ and

$$
h(z, \varsigma) \prec \prec p(z, \varsigma)+\frac{1}{\gamma} z p_{z}^{\prime}(z, \varsigma), \quad(z \in \mathbb{U}, \varsigma \in \overline{\mathbb{U}}),
$$

then

$$
q(z, \varsigma) \prec \prec p(z, \varsigma), \quad(z \in \mathbb{U}, \varsigma \in \overline{\mathbb{U}}),
$$

where $q(z, \varsigma)=\gamma z^{-\gamma} \int_{0}^{z} h(t, \varsigma) t^{\gamma-1} d t$ is convex and it is the best subordinant of (3).

Definition 3 [3]. For $f \in \mathcal{A}_{\varsigma}, m \in N_{0}=N \cup\{0\}, \alpha, \delta \geq 0, \mu, \lambda, \beta>0$ and $\alpha \neq \lambda$, the generalized differential operator $A_{\mu, \lambda, \delta}^{m}(\alpha, \beta): \mathcal{A}_{\varsigma} \rightarrow \mathcal{A}_{\varsigma}$ is defined by

(4) $A_{\mu, \lambda, \delta}^{m}(\alpha, \beta) f(z, \varsigma)=z+\sum_{k=2}^{\infty}\left[1+\frac{(k-1)[(\lambda-\alpha) \beta+k \delta]}{\mu+\lambda}\right]^{m} a_{k}(\varsigma) z^{k}$,

Here, we would point out some of the special cases of the operator defined by (4) can be found in $[1,2,4,6,10,11]$.

In recent years, many authors obtained various interesting results associated with strong differential subordination and superordination for example (see $[5,7,12])$. 


\section{Key Lemma and Main Results}

Before stating and proving our main results, we first establish the following identities involving the operator defined by (4):

Lemma 3 If $f \in \mathcal{A}_{\varsigma}, m \in N_{0}=N \cup\{0\}, \alpha, \delta \geq 0, \mu, \lambda, \beta>0, \alpha \neq \lambda$ and the operator $A_{\mu, \lambda, \delta}^{m}(\alpha, \beta)$ be defined by (4), then

$$
\begin{aligned}
z\left(A_{\mu, \lambda, \delta}^{m}(\alpha, \beta) f(z, \varsigma)\right)_{z}^{\prime} & =\frac{\mu+\lambda}{(\lambda-\alpha) \beta+k \delta} A_{\mu, \lambda, \delta}^{m+1}(\alpha, \beta) f(z, \varsigma) \\
& +\left(1-\frac{\mu+\lambda}{(\lambda-\alpha) \beta+k \delta}\right) A_{\mu, \lambda, \delta}^{m}(\alpha, \beta) f(z, \varsigma) .
\end{aligned}
$$

Proof. In the light of (4), we find that

$$
\begin{aligned}
& \frac{\mu+\lambda}{(\lambda-\alpha) \beta+k \delta} A_{\mu, \lambda, \delta}^{m+1}(\alpha, \beta) f(z, \varsigma) \\
& +\left(1-\frac{\mu+\lambda}{(\lambda-\alpha) \beta+k \delta}\right) A_{\mu, \lambda, \delta}^{m}(\alpha, \beta) f(z, \varsigma) \\
& =z+\sum_{k=2}^{\infty} \frac{\mu+\lambda}{(\lambda-\alpha) \beta+k \delta}\left[1+\frac{(k-1)[(\lambda-\alpha) \beta+k \delta]]}{\mu+\lambda}\right]^{m+1} a_{k}(\varsigma) z^{k} \\
& +\sum_{k=2}^{\infty}\left(1-\frac{\mu+\lambda}{(\lambda-\alpha) \beta+k \delta}\right)\left[1+\frac{(k-1)[(\lambda-\alpha) \beta+k \delta]}{\mu+\lambda}\right]^{m} a_{k}(\varsigma) z^{k} \\
& =z+\sum_{k=2}^{\infty}\left(\frac{\mu+\lambda}{(\lambda-\alpha) \beta+k \delta} \frac{\mu+\lambda+(k-1)[(\lambda-\alpha) \beta+k \delta]}{\mu+\lambda}\right. \\
& \left.+\frac{(\lambda-\alpha) \beta+k \delta-\mu-\lambda}{(\lambda-\alpha) \beta+k \delta}\right)\left[1+\frac{(k-1)[(\lambda-\alpha) \beta+k \delta]}{\mu+\lambda}\right]^{m} a_{k}(\varsigma) z^{k} \\
& \quad=z+\sum_{k=2}^{\infty} k\left[1+\frac{(k-1)[(\lambda-\alpha) \beta+k \delta]}{\mu+\lambda}\right]^{m} a_{k}(\varsigma) z^{k},
\end{aligned}
$$

which establishes the identity (5).

Definition 4 Assume that $\psi(z, \varsigma)$ be an holomorphic function in $\mathbb{U} \times \overline{\mathbb{U}}$ with $\psi(0, \varsigma)=1$ for all $\varsigma \in \overline{\mathbb{U}}$ and $\eta, \mu, \lambda, \beta>0, \alpha, \delta \geq 0, \alpha \neq \lambda, m \in N_{0} . A$ function $f \in \mathcal{A}_{\varsigma}$ is called in the class $V(\eta, \mu, \lambda, \delta, \alpha, \beta, m ; \psi)$ if the following 
strong differential subordination is satisfied:

$$
\begin{aligned}
& \frac{1}{z}\left[\left(1-\frac{\eta(\mu+\lambda)}{(\lambda-\alpha) \beta+k \delta}\right) A_{\mu, \lambda, \delta}^{m}(\alpha, \beta) f(z, \varsigma)\right. \\
& \left.+\frac{\eta(\mu+\lambda)}{(\lambda-\alpha) \beta+k \delta} A_{\mu, \lambda, \delta}^{m+1}(\alpha, \beta) f(z, \varsigma)\right] \prec \prec \psi(z, \varsigma) .
\end{aligned}
$$

A function $f \in \mathcal{A}_{\varsigma}$ is called in the class $W(\eta, \mu, \lambda, \delta, \alpha, \beta, m ; \psi)$ if the following strong differential superordination is satisfied:

$$
\begin{aligned}
\psi(z, \varsigma) \prec & \prec \frac{1}{z}\left[\left(1-\frac{\eta(\mu+\lambda)}{(\lambda-\alpha) \beta+k \delta}\right) A_{\mu, \lambda, \delta}^{m}(\alpha, \beta) f(z, \varsigma)\right. \\
& \left.+\frac{\eta(\mu+\lambda)}{(\lambda-\alpha) \beta+k \delta} A_{\mu, \lambda, \delta}^{m+1}(\alpha, \beta) f(z, \varsigma)\right] .
\end{aligned}
$$

Theorem 1 Suppose that $\psi(z, \varsigma)$ be a convex function in $\mathbb{U} \times \overline{\mathbb{U}}$ with $\psi(0, \varsigma)=$ 1 for all $\varsigma \in \overline{\mathbb{U}}$ and $\eta>0$. If $f \in V(\eta, \mu, \lambda, \delta, \alpha, \beta, m ; \psi)$, then there exists a convex function $q(z, \varsigma)$ such that $q(z, \varsigma) \prec \prec \psi(z, \varsigma)$ and $f \in V(0, \mu, \lambda, \delta, \alpha, \beta, m ; q)$.

Proof. Assume that

$$
\begin{aligned}
p(z, \varsigma) & =\frac{A_{\mu, \lambda, \delta}^{m}(\alpha, \beta) f(z, \varsigma)}{z} \\
& =1+\sum_{k=2}^{\infty}\left[1+\frac{(k-1)[(\lambda-\alpha) \beta+k \delta]}{\mu+\lambda}\right]^{m} a_{k}(\varsigma) z^{k-1} .
\end{aligned}
$$

It is obvious that $p \in \mathcal{H}[1,1, \varsigma]$.

Since $f \in V(\eta, \mu, \lambda, \delta, \alpha, \beta, m ; \psi)$, then we find that

$$
\begin{aligned}
& \frac{1}{z}\left[\left(1-\frac{\eta(\mu+\lambda)}{(\lambda-\alpha) \beta+k \delta}\right) A_{\mu, \lambda, \delta}^{m}(\alpha, \beta) f(z, \varsigma)\right. \\
& \left.+\frac{\eta(\mu+\lambda)}{(\lambda-\alpha) \beta+k \delta} A_{\mu, \lambda, \delta}^{m+1}(\alpha, \beta) f(z, \varsigma)\right] \prec \prec \psi(z, \varsigma) .
\end{aligned}
$$

Now from (5), (6) and (7), it is evident that

$$
\begin{aligned}
& \frac{1}{z}\left[\left(1-\frac{\eta(\mu+\lambda)}{(\lambda-\alpha) \beta+k \delta}\right) A_{\mu, \lambda, \delta}^{m}(\alpha, \beta) f(z, \varsigma)\right. \\
& \left.+\frac{\eta(\mu+\lambda)}{(\lambda-\alpha) \beta+k \delta} A_{\mu, \lambda, \delta}^{m+1}(\alpha, \beta) f(z, \varsigma)\right] \\
& =p(z, \varsigma)+\eta z p_{z}^{\prime}(z, \varsigma) \prec \prec \psi(z, \varsigma) .
\end{aligned}
$$


By making use of Lemma 1 with $\gamma=\frac{1}{\eta}$, we deduce that

$$
p(z, \varsigma) \prec \prec q(z, \varsigma) \prec \prec \psi(z, \varsigma) .
$$

It follows from (6) that

$$
\frac{A_{\mu, \lambda, \delta}^{m}(\alpha, \beta) f(z, \varsigma)}{z} \prec \prec q(z, \varsigma) \prec \prec \psi(z, \varsigma),
$$

where

$$
q(z, \varsigma)=\frac{1}{\eta} z^{-\frac{1}{\eta}} \int_{0}^{z} \psi(t, \varsigma) t^{\frac{1}{\eta}-1} d t
$$

is convex and it is the best dominant.

This shows that $f \in V(0, \mu, \lambda, \delta, \alpha, \beta, m ; q)$ and the proof is completed.

Theorem 2 Suppose that $\psi(z, \varsigma)$ be a convex function in $\mathbb{U} \times \overline{\mathbb{U}}$ with $\psi(0, \varsigma)=$ 1 for all $\varsigma \in \overline{\mathbb{U}}$ and $\eta>0$. If $f \in W(\eta, \mu, \lambda, \delta, \alpha, \beta, m ; \psi), \frac{A_{\mu, \lambda, \delta}^{m}(\alpha, \beta) f(z, \varsigma)}{z} \in$ $\mathcal{H}[1,1, \varsigma] \cap Q_{\varsigma}$ and

$$
\begin{aligned}
& \frac{1}{z}\left[\left(1-\frac{\eta(\mu+\lambda)}{(\lambda-\alpha) \beta+k \delta}\right) A_{\mu, \lambda, \delta}^{m}(\alpha, \beta) f(z, \varsigma)\right. \\
& \left.+\frac{\eta(\mu+\lambda)}{(\lambda-\alpha) \beta+k \delta} A_{\mu, \lambda, \delta}^{m+1}(\alpha, \beta) f(z, \varsigma)\right]
\end{aligned}
$$

is schlicht in $\mathbb{U} \times \overline{\mathbb{U}}$, then there exists a convex function $q(z, \varsigma)$ such that $f \in W(0, \mu, \lambda, \delta, \alpha, \beta, m ; q)$.

Proof. Suppose that the function $p(z, \varsigma)$ be defined by (6). It is evident that $p \in \mathcal{H}[1,1, \varsigma] \cap Q_{\varsigma}$.

After a short calculation and considering $f \in W(\eta, \mu, \lambda, \delta, \alpha, \beta, m ; \psi)$, we can conclude that

$$
\psi(z, \varsigma) \prec \prec p(z, \varsigma)+\eta z p_{z}^{\prime}(z, \varsigma) .
$$

By making use of Lemma 2 with $\gamma=\frac{1}{\eta}$, we obtain

$$
q(z, \varsigma) \prec \prec p(z, \varsigma) .
$$

In view of $(6)$, yields

$$
q(z, \varsigma) \prec \prec \frac{A_{\mu, \lambda, \delta}^{m}(\alpha, \beta) f(z, \varsigma)}{z},
$$


where

$$
q(z, \varsigma)=\frac{1}{\eta} z^{-\frac{1}{\eta}} \int_{0}^{z} \psi(t, \varsigma) t^{\frac{1}{\eta}-1} d t
$$

is convex and it is the best subordinant and the theorem is proved.

If we combine the results of Theorem 1 and Theorem 2, we obtain the following "strong sandwich theorem".

Theorem 3 Let $\psi_{1}(z, \varsigma)$ and $\psi_{2}(z, \varsigma)$ be convex functions in $\mathbb{U} \times \overline{\mathbb{U}}$ with $\psi_{1}(0, \varsigma)=\psi_{2}(0, \varsigma)=1$ for all $\varsigma \in \overline{\mathbb{U}}$ and $\eta>0$. If

$$
\begin{gathered}
f \in V\left(\eta, \mu, \lambda, \delta, \alpha, \beta, m ; \psi_{1}\right) \cap W\left(\eta, \mu, \lambda, \delta, \alpha, \beta, m ; \psi_{2}\right), \\
\frac{A_{\mu, \lambda, \delta}^{m}(\alpha, \beta) f(z, \varsigma)}{z} \in \mathcal{H}[1,1, \varsigma] \cap Q_{\varsigma}
\end{gathered}
$$

and

$$
\begin{aligned}
& \frac{1}{z}\left[\left(1-\frac{\eta(\mu+\lambda)}{(\lambda-\alpha) \beta+k \delta}\right) A_{\mu, \lambda, \delta}^{m}(\alpha, \beta) f(z, \varsigma)\right. \\
& \left.+\frac{\eta(\mu+\lambda)}{(\lambda-\alpha) \beta+k \delta} A_{\mu, \lambda, \delta}^{m+1}(\alpha, \beta) f(z, \varsigma)\right]
\end{aligned}
$$

is schlicht in $\mathbb{U} \times \overline{\mathbb{U}}$, then

$$
f \in V\left(0, \mu, \lambda, \delta, \alpha, \beta, m ; q_{1}\right) \cap W\left(0, \mu, \lambda, \delta, \alpha, \beta, m ; q_{2}\right),
$$

where

$$
q_{1}(z, \varsigma)=\frac{1}{\eta} z^{-\frac{1}{\eta}} \int_{0}^{z} \psi_{1}(t, \varsigma) t^{\frac{1}{\eta}-1} d t
$$

and

$$
q_{2}(z, \varsigma)=\frac{1}{\eta} z^{-\frac{1}{\eta}} \int_{0}^{z} \psi_{2}(t, \varsigma) t^{\frac{1}{\eta}-1} d t .
$$

The functions $q_{1}$ and $q_{2}$ are convex.

\section{References}

[1] A. A. Lupaş, A note on special strong differential superordinations using multiplier transformation, Journal of Computational Analysis and Applications, vol. 17, no. 4, 2014, 746-751. 
[2] F. M. Al-Oboudi, On univalent functions defined by a generalized Sălăgean operator, Int. J. Math. Math. Sci., vol. 27, 2004, 1429-1436.

[3] A. Amourah, M. Darus, Some properties of a new class of univalent functions involving a new generalized differential operator with negative coefficients, Indian J. Sci. Tech., vol. 9, no. 36, 2016, 1-7.

[4] N. E. Cho, T. H. Kim, Multiplier transformations and strongly close-toconvex functions, Bull. Korean Math. Soc., vol. 40, no. 3, 2003, 399-410.

[5] N. E. Cho, O. S. Kwon, H. M. Srivastava, Strong differential subordination and superordination for multivalently meromorphic functions involving the Liu- Srivastava operator, Integral transforms Spec. Funct., vol. 21, no. $8,2010,589-601$.

[6] M. Darus, R. W. Ibrahim, On subclasses for generalized operators of complex order, Far East J. Math. Sci., vol. 33, no. 3, 2009, 299-308.

[7] M. P. Jeyaraman, T. K. Suresh, Strong differential subordination and superordination of analytic functions, J. Math. Anal. Appl., vol. 385, no. $2,2012,854-864$.

[8] S. S. Miller, P. T. Mocanu, Differential Subordinations. Theory and Applications, Series on Monographs and Textbooks in Pure and Applied Mathematics, vol. 225, Marcel Dekker Inc., New York, Basel, 2000.

[9] G. I. Oros, Strong differential superordination, Acta Universitatis Apulensis, vol. 19, 2009, 101-106.

[10] G. St. Sălăgean, Subclasses of univalent functions, Lecture Notes in Math., Springer Verlag, Berlin, vol. 1013, 1983, 362-372.

[11] A. K. Wanas, A. A. Lupaş, On a new strong differential Subordinations and superordinations of analytic functions involving the generalized differential operator, Int. J. Pure Appl. Math., vol. 116, no. 3, 2017, 571-579.

[12] A. K. Wanas, A. H. Majeed, New strong differential subordinations and superordinations of symmetric analytic functions, Int. J. Math. Anal., vol. 11, no. 11, 2017, 543-549. 


\section{Abbas Kareem Wanas}

University of Al-Qadisiyah

College of Science

Department of Mathematics

Diwaniya, Iraq

e-mail: abbas.kareem.w@qu.edu.iq 